Department of Internal Medicine II, University-Hospital Campus Grosshadern,

Ludwig-Maximilians-University of Munich, Munich, Germany

\section{Correspondence to}

Dr Christoph J Auernhammer, Department of Internal Medicine II, University-Hospital Campus Grosshadern, Interdisciplinary Center of Neuroendocrine

Tumours of the

GastroEnteroPancreatic System (GEPNET-KUM),

Ludwig-Maximilians-University of Munich, Marchioninistrasse 15, Munich 81377, Germany; christoph.auernhammer@med. uni-muenchen.de

Published Online First

4 March 2011

\title{
Therapeutic strategies for advanced neuroendocrine carcinomas of jejunum/ileum and pancreatic origin
}

\author{
Christoph J Auernhammer, Burkhard Göke
}

\section{ABSTRACT}

Multimodal treatment options for advanced gastroenteropancreatic neuroendocrine tumours (NET) of jejunum/ileum and of pancreatic origin are reviewed. Current topics being discussed are: European Neuroendocrine Tumour Society 2006/7, American Joint Cancer Committee/Union Internationale Contre le Cancer 2009 and WHO 2010 recommendations for grading and staging of NET; surgery of the primary tumour in distant metastasised disease; surgery of metastatic liver disease and impact on survival; somatostatin analogues for symptom control and for tumour control; selective internal radiation therapy with 90Y-microspheres as novel local ablative therapy in liver metastases; peptide receptor radionuclide therapy; novel chemotherapy regimens (eg, temozolomide) and novel targeted therapies (eg, sunitinib and everolimus).

Neuroendocrine tumours (NET) of the gastroenteropancreatic system can originate from every part of the gastroenteropancreatic system and have been classified during the past decade as benign well-differentiated NET, malignant well-differentiated neuroendocrine carcinomas (NEC) and poorly differentiated NEC. ${ }^{1-3}$ Specific hormone hypersecretion-related functional syndromes (eg, carcinoid syndrome, insulinoma, gastrinoma and others) are only present in approximately $20-30 \%$ of all patients with NET. ${ }^{4-6}$

National databases ${ }^{6-8}$ and tertiary referral centres ${ }^{5}$ have shown that the primary tumour location of NET of the gastroenteropancreatic system is frequently in the jeunum/ileum or in the pancreas with $16-29 \%$ or $31-34 \%$, respectively. NET in the jeunum/ileum and in the pancreas have been shown to present at diagnosis with regional or distant metastasised disease in $71-89 \%$ and $54-86 \%$ of cases, respectively. ${ }^{5-7}$ Therefore, from an oncological point of view, NET of the jejunum/ ileum and of the pancreas are the most common primary tumour entities of NEC within the gastroenteropancreatic system. ${ }^{5-7}$

\section{STAGING, GRADING AND PROGNOSTIC FACTORS}

Numerous prognostic markers for gastroenteropancreatic NET have been described. ${ }^{9}{ }^{10}$ In the clinical routine, important prognostic markers of gastroenteropancreatic NET are primary tumour localisation, tumour grading including proliferation index Ki-67 (Mib-1), tumour staging and pattern of hepatic tumour load.

NET of the pancreas have a poorer prognosis than NET of the jejunum/ileum. ${ }^{57}$ NET of the pancreas and NET of the jejunum/ileum accumulate different patterns of genetic defects during tumour transformation. They must thus be regarded as different tumour entities. ${ }^{11}$ Both tumour entities differ not only in their tumour biology but also in their clinical behaviour and prognosis. In a total of 35618 NET, registered in the Surveillance, Epidemiology and End Results (SEER) Programme from 1973 to 2004, the median survival rate was 88 months in NET of the jejunum/ileum versus 42 months in pancreatic NET. ${ }^{7}$ Regional metastatic disease was present in $41 \%$ of all NET of the jejunum/ileum and in $22 \%$ of all pancreatic NET. However, distant metastatic disease was present in $30 \%$ of all NET of the jejunum/ileum and in $64 \%$ of all pancreatic NET. In NET of the jejunum/ileum and in pancreatic NET 5-year survival rates were $71 \%$ versus $62 \%$ in regional metastatic disease and $54 \%$ versus $27 \%$ in distant metastatic disease. ${ }^{7}$ In a single centre retrospective analysis of 104 ileum and 98 pancreatic NET, Pape et $a l^{5}$ reported a 5 -year survival rate of $90 \%$ versus $69 \%$ and a 10 -year survival rate of $63 \%$ versus $62 \%$. The beneficial impact of large volume centres on survival outcomes still needs to be assessed. Still this may explain the different data from national registries versus single large volume centre series. ${ }^{5}$ Bilimoria et al $^{12}$ reported data from the National Cancer Data Base (1985-2004) of 3851 patients who underwent surgery for pancreatic NET. Overall survival rate was $59.3 \%$ at 5 years and $37.7 \%$ at 10 years. ${ }^{12}$ In a multivariate Cox proportional hazard model, highly significant negative prognostic factors were high-grade histology (HR 2.03, 95\% CI 1.41 to 2.92, $\mathrm{p}<0.0001$ ), liver metastases (HR 1.71, 95\% CI 1.36 to $2.14, \mathrm{p}<0.0001)$ and other distant metastases (HR 3.78, 95\% CI 1.51 to 9.43, p<0.0001). ${ }^{12}$ These data thus reveal that NET have a poorer prognosis than previously assumed and implied by the historic term 'carcinoid'. 12

The historic term 'carcinoid' has been replaced by the WHO classification 2000 for gastroenteropancreatic NET, which classifies tumours according to their primary tumour site and discriminates between benign well-differentiated endocrine tumours and malignant well-differentiated endocrine carcinomas and poorly differentiated endocrine carcinomas. ${ }^{1-3}$ The WHO classification 


\section{Summary}

\section{Current standards}

- Recent comprehensive guidelines for the diagnosis and treatment of gastroenteropancreatic NET have been published by the European Neuroendocrine Tumour Society (ENETS), the National Comprehensive Cancer Network (NCCN) and the North American Neuroendocrine Tumour Society (NANETS).

- Surgery is the only curative option for NEC. Surgery of liver metastases and debulking surgery are essential in NEC.

- Somatostatin analogues are important for symptom control of functionally active NEC.

- Peptide receptor radionuclide therapy with 90Yttrium-DOTA-TOC, 90YttriumDOTA-TATE and 177Lutetium-DOTA-TATE is a promising strategy in distant metastases of NEC.

Novel findings and future perspectives

- Somatostatin analogues may be considered for antiproliferative treatment in well-differentiated functional and non-functional NET of the jejunum/ileum.

- Temozolomide plus capecitabine is a novel promising chemotherapy protocol in pancreatic NEC, which merits further evaluation.

- Selective internal radiation therapy with 90Y-microspheres is a novel local ablative strategy in disseminated liver metastases.

- Novel targeted therapies with the mammalian target of rapamycin (mTOR) inhibitor everolimus or the multikinase inhibitor sunitinib, both improve progression-free survival in NEC of the pancreas. Sunitinib has been approved by the European Medicines Agency (EMA) for this indication in 11/2010. Everolimus may also be expected to get approved in the near future.

- Neoadjuvant and adjuvant therapeutic strategies for liver surgery of hepatic metastasised NEC are urgently needed. Further research should address this issue.

system 2000 envolved stage-related information to discriminate between well-differentiated endocrine tumours and well-differentiated endocrine carcinomas. $^{1-3}$ The novel WHO classification $2010^{13} 14$ assumes neuroendocrine neoplasms as a category to be potentially malignant. The novel histopathological grading system discriminates NET G1 (carcinoid), NET G2, and NEC of small cell and large cell type. In order to address the dignity and prognosis of a neuroendocrine neoplasm, the novel WHO classification $2010^{13} 14$ combines histopathological grading and tumour site-specific tumour-node-metastasis (TNM) staging. For example, a local or distant metastasised NET G1/G2 according to WHO classification 2010, would have been classified as well-differentiated NEC according to WHO classification 2000. On the other hand, a small cell or large cell NEC according to WHO classification 2010 would have been classified as poorly differentiated NEC according to WHO classification 2000. To avoid misunderstandings in clinical routine, medical reports should name the WHO classification system and the TNM classification system used. The nomenclature of the WHO classification system 2000 may still be used in addition to the WHO classification system 2010. ${ }^{13}{ }^{14}$ Nevertheless, in the current literature, NET of the jejunum/ileum are still often termed 'midgut carcinoid', while NET of the pancreas are still often named 'islet cell tumour' or the WHO classification 2000 has been used. Therefore, in this review the term 'neuroendocrine carcinoma' is still used to refer to well-differentiated endocrine carcinomas in accordance with the literature cited. The ENETS classification 2006/7, ${ }^{15} 16$ the 7 th American Joint Cancer Committee/Union Internationale Contre le Cancer (AJCC/UICC) classification 2009 $17-20$ and the current WHO classification $2010^{31319}$ all equally define a grading system based upon the proliferation index Ki-67 and/or mitotic index with low-grade G1 tumours (Ki-67 $\leq 2 \%$, mitotic count $<2$ per 10 high-power field; HPF), intermediate-grade G2 tumours (Ki-67 $3-20 \%$, mitotic count $2-20$ per $10 \mathrm{HPF}$ ) and highgrade G3 tumours (Ki-67 $>20 \%$, mitotic count $>20 \%$ ). These three-tiered grading systems including the proliferation index Ki-67 (Mib-1) and/ or the mitotic index have been demonstrated to be high impact prognostic markers for NET. ${ }^{5} 12$ 21-25 For example, in a series of 202 upper gastrointestinal NET Pape et $a l^{23}$ demonstrated that G1 tumours (Ki-67 $\leq 2 \%$ ), G2 tumours (Ki-67 3-20\%) and G3 tumours (Ki-67 >20\%) showed a 5 -year survival rate of $95.7 \%, 73.4 \%$ and $27.7 \%$, respectively. ${ }^{23}$ The prognostic importance of histopathological grading has also been demonstrated in a series of 324 patients with pancreatic NET. In a multivariate analysis, significantly negative prognostic markers were a proliferation index Ki-67 greater than $2 \%$ (HR 5.2, CI 95\% 1.8 to $15.0, \mathrm{p}=0.002$ ) or histologically poorly differentiated NEC (HR 9.9, CI 95\% 2.9 to $34.0, \mathrm{p}<0.001){ }^{21}$ Therefore, histopathological grading including proliferation index $\mathrm{Ki}-67$ is essential for prognosis and is highly recommended for the clinical routine. ${ }^{26} 27$

Several retrospective analyses from the Surveillance, Epidemiology and End Results (SEER) database and suggestions for a TNM classification system for gastroenteropancreatic NET have been published by Landry and colleagues. ${ }^{28-33}$ Two official TNM classification systems for gastroenteropancreatic NET have recently been provided by ENETS 2006/7 $7^{15} 16$ (http://www.neuroendocrine.net/) and by AJCC/UICC 7th edition (http:// www.uicc.org/resources/tnm). ${ }^{17} 18 \quad 20$ These two TNM classification systems differ specifically in the $\mathrm{T}$ stages for NET of the appendix and of the pancreas. $^{18} 2634$ Pathologists as well as clinicians should be aware of this circumstance and medical reports should name the TNM classification system being used. For the ENETS 2006/7 TNM classification $^{15} 16$ of pancreatic NET, several large series have already demonstrated a good prognostic discrimination, ${ }^{12} 21-2334$ which might be even further improved in the future. ${ }^{34}$

Distant metastatic disease is without doubt a poor prognostic factor. The liver most frequently resembles a metastatic dissemination site..$^{524356}$ Furthermore, the pattern and extent of hepatic tumour load help to determine different treatment options and prognosis in patients with liver metastasis. $^{35}$ 37-39 In 118 patients with distant metastasis from well-differentiated gastroenteropancreatic NEC, predictors of survival were the number of initial diagnosed liver metastasis 
(HR 3.4, 95\% CI 1.4 to 8.3, $\mathrm{p}=0.01$ ) and initial surgery (HR $0.3,95 \%$ CI 0.1 to $0.8, p=0.01$ ). ${ }^{37}$ In another series of 119 patients with hepatic metastases of NEC, ${ }^{38} 5$ and 10 -year survival rates were $100 \%$ both in the subgroup with single liver metastasis, versus $84 \%$ and $75 \%$ in the subgroup with isolated metastatic bulk accompanied by smaller deposits, versus $51 \%$ and $29 \%$ in the subgroup with disseminated metastatic spread. ${ }^{38}$ Interestingly, in the PROMID study a greater than $10 \%$ metastatic tumour load of the liver was defined as a negative prognostic factor for tumour response to biotherapy in 85 patients with nonresectable metastasised NEC. ${ }^{35}$ In 310 patients with non-resectable NEC, treated with 177Lutetium-DOTA-Tyr3-octreotate (177Lu-DOTA-TATE), extensive liver involvement was found to be a negative prognostic factor for survival after peptide receptor radionuclide therapy (PRRT). ${ }^{39}$

The prognostic impact of clinically functional versus non-functional tumours is controversial. ${ }^{12} 2122 \quad 36$ While some studies found no prognostic effect of the functional status in NET of the pancreas, ${ }^{21} 36$ others reported non-functional tumours $^{22}$ or vice versa functional tumours ${ }^{12}$ as predictors of improved survival.

\section{SURGERY OF THE PRIMARY IN LOCAL DISEASE AND IN METASTASISED DISEASE}

Surgery of NET is the sole curative option. Surgery of the primary tumour may be also advantageous in well-differentiated NEC with local lymph node and distant liver metastasis according to concepts of 'debulking' for tumour mass reduction.

In NEC of the midgut, resection of the primary has been reported to be an independent positive predictor of survival. ${ }^{40} 41$ In a single centre study series by Hellman et a l $^{41}$ in 314 patients with midgut carcinoid tumours, the median survival was 7.4 years in the primary resected group versus 4.0 years in the primary non-resected group. In the multicentre study UKINETS, ${ }^{40}$ with 360 patients with midgut carcinoid tumours, resection of the primary was an independent positive predictor of survival. In contrast, no significant survival benefit of primary tumour resection was reported in two other series of metastatic NEC. ${ }^{24} 35$ Following total surgery of midgut NET, tumour recurrence has been observed in $59 \%$, with a median time to recurrence of 32 months. ${ }^{42}$ Resection of the primary tumour with mesenteric lymph nodes due to oncological criteria is also recommended in the presence of unresectable liver metastasis in patients with welldifferentiated NEC of the jejunum/ileum by guidelines from ENETS 2008, ${ }^{43}$ NCCN $2009^{44}$ and NANETS 2010. ${ }^{45}$ The rationale for this recommendation is to avoid desmoplastic reaction with bowel obstruction and/or bowel ischaemia reaching a situation in which only the liver metastases need further treatment.

In NEC of the pancreas, aggressive surgery of the primary has also been reported not only in local but also in distant disease with liver metastasis. ${ }^{46}$ In a retrospective survival analysis of 728 patients with pancreatic NET from the SEER database (1988-2002), the median survival was 114 months in the resected group versus 35 months in a nonresected group, with an adjusted HR of $0.48 .{ }^{47}$ This advantage in median survival was not only significant in localised disease, but also in regional metastasised disease (resection 129 months vs resection recommended/not resected 64 months) and distant metastasised disease (resection 60 months vs resection recommended/not resected 31 months). ${ }^{47}$ Data from the National Cancer Data Base (1985-2004) on 3851 patients undergoing surgery for pancreatic NET showed no significant effect of primary tumour size, nodal status, resection margins or surgical procedure in a multivariate Cox proportional hazard model. ${ }^{12}$ However, a highly significant negative prognostic factor was age 55-75 years (HR 1.57, 95\% CI 1.28 to 1.91 , $\mathrm{p}<0.0001$ ) and age greater than 75 years (HR 3.04, $95 \%$ CI 2.17 to $4.25, \mathrm{p}<0.0001) .{ }^{12}$ The patient's age should thus be considered in clinical decisionmaking when evaluating pancreatic surgery. In several large surgical series of NEC of the pancreas, morbidity and mortality ranged between $27-43 \%$ and $0-3.4 \%$, while 5 -year survival rates for malignant tumours were $49-77 \% .^{22} 46 \quad 48-51$ A postoperative complication rate of $29.6 \%$ has been reported in a nationwide inpatient sample survey encompassing 463 patients after pancreatic surgery from 1998 to $2006 .{ }^{52}$ Risk factors for complications were a high Charlson comorbidity score as well as Whipple operation or total pancreatectomy. ${ }^{52}$ The potential benefits reported in a single uncontrolled series $^{47}$ may thus have been biased by patient preselection and also need to be weighed up against the morbidity and mortality of these surgical procedures. Whereas for pancreatic NEC with resectable liver metastases complete resection of the primary and the liver metastases is recommended by various guidelines, ${ }^{44} 53$ a primary tumour resection of the pancreas is not recommended in current guidelines for patients with unresectable disseminated liver metastasis. ${ }^{53} 54$

\section{SURGERY OF LIVER METASTASES}

Surgery of synchronous or metachronous liver metastases in well-differentiated NEC is recommended when complete resection or debulking more than $90 \%$ of the tumour load seems feasible. ${ }^{55}$ In patients with extended bilobar liver metastases, the feasibility of a two-step resection should be evaluated in an experienced centre. ${ }^{55}{ }^{56}$ Using the two-step approach non-anatomical limited resection of liver metastases in the left liver plus right portal vein ligation is followed consecutively by right hemihepatectomy after an adequate hypertrophy of the left liver has been reached. ${ }^{55} 56$ In a large retrospective, multicentre series of 339 patients with NET liver metastases following surgery of liver metastases the 5 and 10-year survival rates were $74 \%$ and $51 \%,{ }^{57}$ indicating a prolonged survival. However, 5-year tumour recurrence in several series has been reported in up to $94 \%$ to $97 \%,{ }^{58}$ indicating a non-curative 
approach in most cases. Complete and/or debulking cytoreductive surgery of liver metastases has also been reported as a positive predictor of survival in several other retrospective uncontrolled series. ${ }^{40} 5960$ However, so far, there is no evidence from randomised studies comparing surgery of liver metastases versus other treatments in patients with liver metastases from NET. ${ }^{61}{ }^{62}$ Sarmiento and Que ${ }^{60}$ reported in a large series of 170 patients with NEC after surgery of liver metastases (44\% curative resection, $56 \%$ debulking $>90 \%$ of tumour load) a 5 -year survival rate of $61 \%$ and, nevertheless, a 5 -year tumour recurrence rate of $84 \%$. Similarly, in a single-centre series of 41 patients with hepatic metastasised NEC, tumour recurrence following liver surgery was reported in $78 \%$ of cases after a median of 19 months. ${ }^{58}$ Osborne et al ${ }^{59}$ compared in another series of 120 patients surgery of liver metastases $(60 \%$ curative resection, $40 \%$ palliative cytoreduction) versus transarterial embolisation (TAE), with a mean overall survival of $43 \pm 26$ months versus $24 \pm 16$ months.

At present only single case reports or small uncontrolled series describe neo-adjuvant strategies with radio-receptor (90)Y-DOTATOC therapy, ${ }^{63}$ resin (90)Y-microspheres radio-embolisation ${ }^{64}$ or chemotherapy for downsizing hepatic metastasis of NEC before liver surgery. Neo-adjuvant strategies are not yet established in liver surgery of NEC. Adjuvant chemotherapy after resection of neuroendocrine liver metastasis is also not established. ${ }^{55}$ Further controlled clinical studies are clearly needed.

\section{RADIOFREQUENCY ABLATION AND TRANSARTERIAL CHEMOEMBOLISATION}

When surgery seems unfavourable, radiofrequency ablation (RFA) of liver metastasis in NEC may be considered in metastases of fewer than five lesions and metastases with a size less than $5 \mathrm{~cm} .{ }^{66}$ RFA results in a symptomatic response with a better control of functional syndromes due to specific hormone excess in approximately $70-80 \%{ }^{67-69}$ and a duration of symptom control of 6-24 months. ${ }^{67} 69$ Local tumour recurrence following RFA occurred in only $6 \%$ of NEC, ${ }^{68-70}$ while local tumour recurrence tended to occur more often in metastases with a higher Ki-67. ${ }^{68}$ The local recurrence rate following RFA of liver metastasis is dependent on the respective tumour type, as has been reported in a large series of 1032 unresectable liver tumours. Local recurrence rates have been reported as follows: $6 \%$ in NEC; $18 \%$ in hepatocellular carcinomas; and $34 \%$ in colorectal cancer. ${ }^{70}$ Further important prognostic factors for local tumour recurrence following RFA of liver metastases are tumour size, ablation margin, and blood vessel proximity. ${ }^{70}$ No controlled data on overall survival following RFA and comparison of RFA with other local ablative methods are currently available. Side-effects and complications of RFA include pain, bile leakage, liver abscess, intraabdominal haemorrhage, pneumothorax and pleural effusion. ${ }^{69} 7172$
TAE and transarterial chemo-embolisation (TACE) may be discussed for patients with liver metastasis in cases of NEC for all inoperable disseminated hepatic metastases. TAE and/or TACE of liver metastasis in NEC results in morphological response of the metastases with mostly partial tumour regression in approximately $50 \%$, a biochemical response of $50-90 \%$, and symptomatic response with a control of functional syndromes in $60-95 \%$ of cases, as has been extensively reviewed. ${ }^{66} 717374$ Arterial phase enhancement is a positive predictive factor of tumour response. $^{75}$ TAE and TACE seem to be equally efficacious in the treatment of liver metastasis in NEC. ${ }^{6673} 76$ While many different protocols have been reported in uncontrolled series for TAE and/or TACE, ${ }^{66} 7174$ no single protocol shows confirmed superiority. Most centres recommend repeated sessions of TAE or TACE. ${ }^{66}$ A biochemical response measured by a reduction of urinary 5-hydroxyindolic acetic acid (5-HIAA) and serum chromogranin A levels after TAE seems to correlate positively with prolonged survival in a recent series of 107 patients with liver metastasis of midgut carcinoid tumours. ${ }^{77}$ A common side-effect of TAE and/ or TACE is a post-embolisation syndrome with abdominal pain, nausea, vomiting, fever and an increase in hepatic transaminases. ${ }^{71} 7475$ Severe but rare complications are liver failure, cholecystitis, gastric ulcers and bleeding, liver abscess or carcinoid crisis. ${ }^{717475}$ The incidence of severe complications is minimised by superselective catheterisation, embolisation of a limited number of liver segments during a single intervention in cases with high tumour load of the liver and pretreatment with a somatostatin analogue in functionally active tumours. $^{71} 7475$ Current clinical phase II trials are investigating drug-eluting beads with doxorubicin for TACE in NEC ${ }^{78} 79$ (http://ClinicalTrials. gov/ identifier: NCT00730483) and a combination of TACE with the multikinase-inhibitor sunitinib in NEC (http://ClinicalTrials.gov/ identifier: NCT00434109). A combination of TAE or TACE with inhibitors targeting angiogenesis or receptorassociated kinases seems an attractive strategy as embolisation of tumour tissue might cause a flairup of different growth factors including vascular endothelial growth factor (VEGF).

\section{RADIO-EMBOLISATION WITH 9OY-MICROSPHERES}

Selective internal radiation therapy (SIRT) by radio-embolisation of liver metastases with radioactive 90Y-resin microspheres (SIR-Sphere; SIRTEX Medical, Wilmington, USA) or 90Y-glass microspheres (TheraSphere; MDS Nordion, Ottawa, Canada) is currently employed to treat inoperable disseminated hepatic tumours in patients with hepatocellular carcinoma, colon cancer, breast cancer and NEC. SIR-Sphere microspheres are $20-60 \mu \mathrm{m}$ in diameter and the radioactivity load is only approximately $50 \mathrm{~Bq}$ per single microsphere. Approximately $30-60 \times 10^{6}$ SIR-Sphere microspheres are implanted and create a profound embolisation effect. TheraSphere microspheres are 
also $20-30 \mu \mathrm{m}$ in diameter, but the radioactivity load is much higher with approximately $2500 \mathrm{~Bq}$ per single microsphere. As significantly lower numbers of TheraSphere microspheres are required to reach equivalent radiation dosages, the embolisation effect is only minor. SIR-Sphere is approved as a medical device for the treatment of liver metastasis from colon carcinoma in the USA, Australia and Europe. TheraSphere is approved as a medical device for treatment in patients with unresectable hepatocellular carcinoma in the USA, Canada and Europe. Both applications are expensive and reimbursement varies regionally. Kennedy et $a l^{80}$ reported in a large multicentre series of 148 patients with liver metastases of NEC, SIRT by radio-embolisation with 90 Y-resin microspheres (SIR-Sphere) to deliver a median activity of $1.14 \mathrm{GBq}$ (0.33-3.30 GBq). Within 3 months, a complete tumour response was found in $2.7 \%$, a partial response in $60.5 \%$, stable disease in $22.7 \%$ and progressive disease in $4.9 \%{ }^{80}$ The median survival was 70 months. ${ }^{80}$ Using SIRT with 90Yresin microspheres in a smaller series of 34 patients with NEC, King et al ${ }^{81}$ reported symptomatic responses in approximately $50 \%$ of all patients and radiological tumour response in 50\% (complete response in $18 \%$ and partial response in $32 \%$ ). A biochemical response of the tumour marker chromogranin A was found in approximately $40 \%{ }^{81}$ The mean overall survival was $29 \pm 3$ months. ${ }^{81}$ Rhee et al ${ }^{82}$ reported in another series of 42 patients with NEC treated with radioactive 90Y-resin microspheres (SIR-Sphere) or 90Y-glass microspheres (TheraSphere) a tumour response within 6 months of approximately 90\% (partial response in $50-54 \%$ and stable disease in $42-44 \%$ ) and a projected median survival of $22-28$ months. Cao et $a l^{83}$ reported in a recent series of 51 patients with NEC treated with SIRT 11\% complete response, $27 \%$ partial response, $27 \%$ stable disease and $33 \%$ progressive disease. Median survival in that series was 36 months. ${ }^{83}$ Similarly, Kalinowki et a l $^{84}$ presented in a small series of nine patients with NEC after radioactive 90Y-resin microspheres (SIR-Sphere) a tumour response within 3 months of $66 \%$ partial response and $33 \%$ stable disease, with an estimated time to progression (TTP) of 11 months. Well-differentiated NEC, low hepatic tumour burden, no extrahepatic disease and partial/ complete response to SIRT are prognostic factors associated with improved survival; ${ }^{83} 85$ thus these parameters may be useful for appropriate patient selection. Clinicians should be aware of specific complications of radio-embolisation with 90Ymicrospheres, such as radiation-induced liver disease, ${ }^{86}$ hepatic abscess, ${ }^{87}$ arterial shunting to the lung, radiation microsphere-induced gastric ulceration and bleeding, acute cholecystitis or acute pancreatitis. $^{88} 89$

So far, SIRT with 90Y-microspheres demonstrates radiological tumour response rates and symptomatic response rates within the range of non-radioactive embolisation techniques such as TACE and TAE in NEC. Still, the superiority of SIRT versus
TACE/TAE in NEC may only be assumed based upon theoretical considerations. A significantly improved tumour response has only been published in a single study in hepatocellular carcinoma. ${ }^{90}$ Controlled studies of SIRT versus TACE/TAE are needed in NEC.

\section{PEPTIDE RECEPTOR RADIONUCLIDE THERAPY}

PRRT of NEC has already been extensively reviewed. ${ }^{91-95}$ PRRT is currently based on labelled somatostatin analogues with high affinity to the somatostatin receptor subtype 2, being expressed by $80-95 \%$ of all NET. The currently most frequently used radiopharmaceuticals for PRRT of NEC are 90Yttrium-DOTA-Tyr3-octreotide (90YDOTA-TOC), 90Yttrium-DOTA-Tyr3-octreotate (90Y-DOTA-TATE), and 177Lutetium-DOTA-Tyr3octreotate (177Lu-DOTA-TATE). ${ }^{91-94}$

Adequate tumour uptake in 111In-DTPA-octreotide (111In-pentetreotide) scintigraphy (Octreoscan; Covidien - Mallinckrodt Imaging, Hazelwood, MO 63042, USA) is an essential prerequisite when considering PRRT as a therapeutic option. ${ }^{96}$ Novel somatostatin-based positron emission tomography (PET) imaging techniques such as $68 \mathrm{Ga}$ -

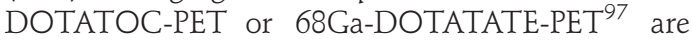
also increasingly used to evaluate adequate tumour uptake before PRRT. Somatostatin receptor subtype 2 immunohistochemistry only modestly correlates with the in-vivo standardised uptake values (SUV) in diagnostic 68Ga-DOTATOC-PET ${ }^{98}$ and $68 \mathrm{Ga}-$ DOTATATE-PET. ${ }^{99}$ A pre-therapeutic high intensity of tumour uptake in 111In-DTPA-octreotide scintigraphy (Octreoscan) $)^{3995}$ seems to be a positive predictor of tumour remission in PRRT. Similarly, significantly higher SUV max $_{\text {ax }}$ of NET in pretherapeutic 68Ga-DOTATATE-PET has been observed in responders versus non-responders to PRRT. ${ }^{100}$ Even 111In-DTPA-octreotide scintigraphy (OctreoScan) negative tumours frequently still demonstrate specific uptake in 68Ga-DOTATATEPET $^{101}$ and thus finally might qualify for PRRT. The potential value of these specific PET/CT techniques as a functional imaging modality to predict early tumour response in the follow-up after PRRT is not confirmed ${ }^{102}$ and still needs further studies. ${ }^{100}$

Preclinical data providing important insights into the principles of PRRT have been published with human midgut GOT1 tumours in a mouse xenograft model. ${ }^{103} 104$ Several uncontrolled clinical studies investigating PRRT with 90Y-DOTATOC, $^{93}$ 105-107 90Y-DOTA-TATE ${ }^{93} 108$ and 177LuDOTA-TATE ${ }^{39}$ have been published (see table 1). Kwekkeboom et al ${ }^{39}$ investigated PRRTwith 177LuDOTA-TATE in the largest published series of 310 patients with NEC. Treatment with 177Lu-DOTATATE caused a complete response in $2 \%$, a partial response in $28 \%$, a minor response in $16 \%$, stable disease in $35 \%$ and progressive disease in $20 \% .{ }^{39}$ The reported median progression-free survival (PFS) rate was 33 months and the median overall survival rate from the start of PRRT was 46 months. ${ }^{39}$ However, in that study only $43 \%$ of all patients had 
Table 1 Peptide receptor radionuclide therapy in well-differentiated neuroendocrine carcinomas

\begin{tabular}{|c|c|c|c|c|c|c|c|}
\hline & n & $\begin{array}{l}\text { Progress at } \\
\text { inclusion (\%) }\end{array}$ & $\begin{array}{l}\text { CR } \\
\text { (\%) }\end{array}$ & $\begin{array}{l}\text { PR } \\
(\%)\end{array}$ & $\begin{array}{l}\text { SD } \\
(\%)\end{array}$ & $\begin{array}{l}\text { PD } \\
(\%)\end{array}$ & $\begin{array}{l}\text { Outcome } \\
\text { (months) }\end{array}$ \\
\hline \multicolumn{8}{|l|}{ 9OY-DOTA-TOC } \\
\hline Otte $1999^{a}$ & 18 & NA & - & 6 & 83 & 11 & NA \\
\hline Waldherr $2001^{b}$ & 37 & 81 & 3 & 24 & 62 & 11 & $\mathrm{TTP}>26$ \\
\hline Waldherr $2002^{c}$ & 37 & 100 & 3 & 19 & 70 & 8 & NA \\
\hline Paganelli $2002^{\mathrm{d}}$ & 87 & 76 & 5 & 23 & 49 & 20 & TTP 14 \\
\hline Bodei $2003^{\mathrm{e}}$ & 20 & NA & - & 30 & 50 & 20 & TTP 10 \\
\hline Valkema $2006^{f}$ & 58 & 76 & - & 9 & 62 & 24 & TTP 29 \\
\hline Forrer $2006^{106}$ & 116 & 93 & 4 & 22 & 62 & 11 & NA \\
\hline Cwikla $2010^{108}$ & 60 & 100 & - & 23 & 70 & 0 & PFS 17 \\
\hline \multicolumn{8}{|l|}{ 9OY-DOTA-TATE } \\
\hline Baum $2004^{g ~ h}$ & 75 & 89 & - & 37 & 52 & 11 & NA \\
\hline Bushnell $2010^{105}$ & 90 & 100 & - & 4 & 70 & 12 & PFS 16 \\
\hline \multicolumn{8}{|l|}{ 177Lu-DOTA-TATE } \\
\hline Kwekkeboom $2008^{39}$ & 310 & 43 & 2 & 28 & 50 & 20 & PFS 33 \\
\hline
\end{tabular}

Adopted from Forrer et $a l^{93}$ and van Essen et al. ${ }^{91}$

$\mathrm{CR}$, complete remission; PFS, progression-free survival; PD, progressive disease; $\mathrm{PR}$, partial remission; $\mathrm{SD}$, stable disease; TTP, time to tumour progression. ticals are produced by local centres following good manufacturing practice (GMP) regulations and reimbursement varies regionally. PRRT is provided by an increasing number of clinical centres in Europe, and PRRT standards for care guidelines have recently been published by ENETS. ${ }^{96}$ No singlecentre individual protocol has confirmed superiority. The maximum tolerated cumulative dose to the kidney has been defined as $27 \mathrm{~Gy} .{ }^{96}$ The administration of amino acid infusions with arginine/lysine is the current standard protocol during PRRT treatments. ${ }^{96}$ This minimises re-uptake of the radiopharmaceutical in the renal tubulus causing a minimised radiation dosage to the kidneys. ${ }^{114} 115$

The side-effects of PRRT might be further minimised in the future by using novel radionuclides such as the alpha-emitter 225 actinum, ${ }^{116}$ or novel nephroprotective agents. ${ }^{114} 117$ One major exclusion criterion for PRRT is insufficient renal function. While a creatinine clearance greater than $40 \mathrm{ml} / \mathrm{min}$ has been suggested as a cut-off for PRRT, $^{96}$ we recommend an even more restrictive cut-off. In patients who do not qualify for PRRT due to impaired renal function or insufficient tumour uptake of radiopharmaceuticals with somatostatin receptor affinity, systemic radiotherapy with 131I-meta-iodobenzylguanidine should be evaluated and considered as an alternative therapeutic option. ${ }^{45}{ }^{118-120}$ Strategies to improve further the therapeutic efficacy of PRRT in future include a combination of PRRT with chemosensitisers such as 5 -fluorouracil, ${ }^{121}$ capecitabine $^{122}$ or temozolomide. ${ }^{123}$

\section{EVALUATION OF TREATMENT EFFICACY IN NEC: LIMITATIONS AND FUTURE REQUIREMENTS}

The efficacy of different therapeutic strategies in NEC is difficult to compare due to the lack of randomised head-to-head studies. The interpretation and comparison of various published study results in NEC are often hampered by small patient numbers and heterogeneous inclusion criteria (eg, time since diagnosis, progressive versus nonprogressive disease, tumour grading, tumour staging, primary tumour location, resected vs nonresected primaries, low vs high hepatic tumour load). Data on treatment outcomes from different studies are thus not directly comparable and must be interpreted with caution. In addition, prospective placebo controlled or standard controlled studies are still missing and are urgently needed in order to demonstrate a beneficial effect on the overall survival and quality of life in patients with NEC for most therapeutic strategies.

In addition, the efficacy of chemotherapeutic regimens in solid tumours has been classically assessed by a change in tumour size using the WHO criteria, the RECIST criteria or the modified RECIST (version 1.1.) criteria. ${ }^{124} 125$ However, most novel anti-angiogenic and molecular targeted therapies in solid tumours cause inhibition of tumour growth rather than regression of tumour size. Therefore, these novel therapies often show low response rates by RECIST criteria, despite their 
clinical benefit. As tumour size regression is not a prerequisite of clinical benefit, criteria solely based on tumour size regression have low prognostic value. Perfusion CT, dynamic contrast-enhanced MRI and FDG-PET response criteria in solid tumours (PERCIST) criteria are emerging novel strategies for functional imaging of solid tumours and prognostic assessment of therapeutic response. $^{125} 126$ Contrast-enhanced CT can also provide more information than the sole tumour size. For example, the Choi criteria (size and density of the tumour) in gastrointestinal stroma tumours $^{127}$ and the MASS criteria (morphology, attenuation, size and structure of the tumour) in renal cell carcinomas ${ }^{128}$ have superior prognostic value during targeted therapy of these tumour entities. Novel radiological criteria for response assessment of NEC have not yet been established. However, novel criteria are urgently needed for prognostic stratification following novel targeted therapies as well as biotherapy, peptide receptor radionuclide therapy or local ablative techniques. Tumour size measurement according to RECIST criteria does not reflect the possible therapeutic benefit of these therapies in NEC. Data based on tumour regression are not always sufficient to compare the efficacy of different therapeutic strategies. Instead, TTP or PFS are alternatively often determined in current studies with novel targeted therapies. TTP or PFS measure the biological activity of cytostatic as well as cytotoxic therapies on tumour burden. They are important parameters to evaluate the biological activity of novel therapies in tumour patients and gain an accelerated approval endpoint. ${ }^{129}$ However, TTP and PFS may not provide reliable evidence that a novel therapy is also of clinical efficacy for the patient. ${ }^{129}$ Clearly, overall survival data are needed. However, multimodal and multisequence treatment strategies in NEC will make it difficult to obtain sufficient study data employing overall survival as the primary endpoint in future. A novel integrated approach is needed to evaluate therapeutic efficacy efficiently. ${ }^{130}$ This approach will have to include functional imaging and biomarkers ${ }^{9131}$ as prognostic indicators, as well as PFS, quality of life $\mathrm{f}^{132}$ and overall survival.

\section{BIOTHERAPY WITH SOMATOSTATIN ANALOGUES AND INTERFERON \\ Somatostatin analogues}

Two somatostatin analogues, octreotide and lanreotide, are currently approved by the European Medicines Agency for symptom control of functionally active $\mathrm{NET}^{133} 134$ as well as for acromegaly. ${ }^{135}$ A current review by Modlin et al ${ }^{134}$ analysed a total of 15 studies including 481 patients with NET. In patients treated with octreotide $(n=261)$, octreotide LAR $(n=122)$, lanreotide $(n=30)$ and lanreotide autogel $(n=185)$, a symptomatic response was seen in $74.2 \%, 77.3 \%, 63.0 \%$ and $67.5 \%$, respectively. ${ }^{134}$ Biochemical response rates were reported with octreotide $(n=315)$, octreotide LAR $(n=73)$, lanreotide $(n=49)$ and lanreotide autogel $(n=333)$ in $50.9 \%, 51.4 \%, 55.0 \%$ and $39.0 \%$, respectively. ${ }^{134}$ These data suggest similar effects of octreotide and lanreotide, while no direct comparison is allowed due to the large heterogeneity of study populations and the lack of a randomised well-controlled head-to-head study. Both compounds are considered equally effective in the control of symptoms and biochemical markers in functioning gastrointestinal NET. ${ }^{43} 133136137$

Antiproliferative actions of somatostatin analogues have been claimed in various preclinical in-vitro and in-vivo models and have been extensively reviewed elsewhere. ${ }^{133} \quad 134$ 138-140 Several retrospective series and uncontrolled studies have suggested an antiproliferative effect of the somatostatin analogues octreotide and lanreotide in highly differentiated NEC. ${ }^{136} 137141$ In a current review analysing a total of 15 studies including 481 patients with NET, ${ }^{134}$ patients treated with octreotide $(n=207)$, octreotide LAR $(n=43)$, lanreotide $(n=87)$ and lanreotide autogel $(\mathrm{n}=220)$ exhibited tumour response $(\mathrm{SD}+\mathrm{PR})$ in $57.4 \%, 69.8 \%, 46.6 \%$ and $64.4 \%$, respectively. ${ }^{134}$ The most common tumour response was stable disease, while partial response was a rare event occurring in only $3-8 \%$ of patients. ${ }^{137}$ Comprehensive reviews by Plockinger and Wiedenmann ${ }^{136}$ and Strosberg and Kvols ${ }^{138}$ compared the antiproliferative effects of octreotide or lanreotide in several clinical trials of patients with documented progressive disease compared with clinical trials of patients without documented progressive disease. Plockinger and Wiedenmann ${ }^{136}$ reported in patients without documented progressive disease (studies $n=8$, patients $n=183$ ) a partial response in $8 \%$ and a stable disease in $60 \%$, while in patients with documented progressive disease (studies $\mathrm{n}=6$, patients $\mathrm{n}=201$ ) a partial response was seen in $3 \%$ and stable disease in $44 \%{ }^{136}$ To summarise, in these non-randomised heterogeneous study populations, tumour stabilisation during therapy with somatostatin analogues was observed in approximately 40-60\%, ${ }^{136-138} 141$ with a reported median duration of stabilisation of 9.5-26 months. ${ }^{136} 138$ Tumour stabilisation rates are lower in patients with previously progressive disease. $^{136} 138$ Spontaneous partial tumour remissions are rare events. ${ }^{136} 137141$ The role of somatostatin analogues for tumour control has been controversially discussed, as these non-randomised studies with heterogenous patient cohorts do not allow an evidence-based judgement on a putative antiproliferative effect of somatostatin analogues.

The placebo-controlled PROMID study has recently demonstrated for the first time in a doubleblind, prospective, randomised trial a superior effect of octreotide LAR against placebo in the control of tumour growth in patients with metastatic neuroendocrine midgut tumours. ${ }^{35}$ The PROMID study enrolled 85 patients with metastatic neuroendocrine midgut tumours. The majority of all patients demonstrated a tumour proliferation index Ki-67 of up to $2 \%$ ( $95.3 \%$ of all patients), a positive Octreoscan $(74.1 \%$ of all patients) and a minor liver tumour load of up to $10 \%$ (75.3\% of all patients). An interim analysis of the PROMID study revealed 
that the median time to tumour progression was 14.3 versus 6.0 months in the octreotide LAR group versus the placebo group, with a HR of 0.34 (95\% CI 0.20 to $0.59 ; p=0.000072) .{ }^{35}$ Stable disease at 6 months of treatment was reported in $66.7 \%$ versus $37.2 \%$ of the patients in the octreotide LAR group versus the placebo group. ${ }^{35}$ Nevertheless, the PROMID data have to be interpreted with caution. ${ }^{142}$ An imbalance in the time to diagnosis in both treatment arms might have favoured the octreotid LAR arm due to the enrolment of patients with more indolent disease. ${ }^{142}$ The PROMID study also does not answer the following clinically important questions: (1) is it advantageous to wait until tumour progression or to treat at initial diagnosis? (2) is there any effect on overall survival regarding early treatment? (3) can these data be extrapolated to patients with NET with Ki-67 greater than $2 \%$ and hepatic tumour load greater than 10\%?. ${ }^{142}$ The NCCN guideline $2009^{44} 143$ and the NANETS guideline $2010^{45}$ discuss the use of octreotid LAR for controlling tumour growth in functioning as well as non-functioning well-differentiated NEC of jejunum/ileum as a possible therapeutic option. However, use of the 'wait and see' strategy for a few months after initial diagnosis of a well-differentiated asymptomatic NEC in order to assess tumour growth dynamics is still another option according to current guidelines. ${ }^{44} 143$ Another phase III, randomised, double-blind, stratified comparative, placebo controlled study of somatostatin analogues for tumour control is still ongoing. That study will assess the effect of lanreotide autogel $120 \mathrm{mg}$ administered every 28 days on tumour PFS in patients with nonfunctioning entero-pancreatic endocrine tumours (http://ClinicalTrials.gov/ identifier: NCT00353496).

Initial therapy with short-acting octreotide 50-200 $\mu \mathrm{g}$ subcutaneously three times a day, followed by subsequent application of slow-release octreotide LAR 10-30 mg deep intramuscularly every 3-4 weeks or lanreotide autogel 60-120 mg deep subcutaneously every $4-6$ weeks is recommended. ${ }^{43} 144$ For the prevention and therapy of a carcinoid crisis short-acting octreotide $50-100 \mu \mathrm{g} / \mathrm{h}$ intravenously is recommended. ${ }^{43} 144$ The safety profile of somatostatin analogues is generally considered advantageous. ${ }^{45} 136144$ Common sideeffects are gastrointestinal discomfort, flatulence and steatorrhea/loose stools. Further side-effects often include asymptomatic sludge in the gallbladder or cholezystolithiasis, but rarely symptomatic cholelithiasis. Exocrine pancreatic insufficiency with malabsorptive steatorrhoea and the need for pancreatic enzyme supplementation, as well as vitamin $\mathrm{D}$ deficiency should be considered during therapy with somatostatin analogues. Bradycardia, hyperglycaemia, hypothyroidism and vitamin B12 deficiency should also be regularly screened for. ${ }^{45} 136$

\section{Interferon alpha}

Interferon alpha is another approved biotherapy for symptom control and antiproliferative effects in
NEC. ${ }^{136} 137145146$ Interferon alpha causes a symptomatic response in $30-70 \%{ }^{45} 136$ however, demonstrating a more delayed onset of response. ${ }^{45136}$ The antiproliferative effects of interferon alpha have been reviewed by Plockinger and Wiedenmann, ${ }^{136}$ analysing a total of 10 studies including 274 patients with NET. Interferon alpha tumour response was reported with stable disease in $70 \%$ and partial response in $11 \%{ }^{136}$ However, these data have to be interpreted with caution as in earlier studies the observation time was too short. ${ }^{136}$ A current phase III trial compared chemotherapy with streptozotocin plus 5-fuorouracil versus interferon-alpha2a (3 MU×3 per week) in 64 patients with progressive advanced metastastic carcinoid tumours of different primary tumour location. ${ }^{146}$ The median PFS (WHO criteria, biochemical progression or death) was 5.5 months for chemotherapy versus 14.1 months for interferon (HR 0.75 (0.41-1.36); not significant). ${ }^{146}$

As result of numerous side-effects interferon alpha is limited in its tolerability, ${ }^{136}$ and therefore is currently only regarded second-line biotherapy compared with somatostatin analogues. ${ }^{43} 53$ Three randomised studies investigating a combination therapy of somatostatin analogues plus interferon alpha versus monotherapy showed a positive nonsignificant trend but failed to demonstrate superiority of the combination therapy. ${ }^{145}$ Therefore, a priori combination therapy of somatostatin analogues with interferon alpha is not recommended. ${ }^{43}$ However, in patients with progressive disease during monotherapy, combination therapy may be an option. ${ }^{43}$ Currently used interferon alpha dose titration regimens and recommended monitoring for side-effects have been described elsewhere. $^{144}$

\section{Novel biotherapeutic agents}

Pasireotide (SOM-230), is a new multireceptor ligand somatostatin analogue currently in clinical development. ${ }^{147}$ While octreotide and lanreotide show binding affinity mostly to the somatostatin receptor subtypes 2 and 5, pasireotide additionally demonstrates significant binding affinities to somatostatin receptor subtypes 1 and $3 .{ }^{147}$ Furthermore, when compared with octreotide, pasireotide demonstrates slightly different signalling effects. In contrast to octreotide inducing somatostatin receptor type 2 internalisation, ${ }^{148}$ pasireotide does not cause somatostatin receptor type 2 internalisation. $^{149-151}$ Pasireotide in comparison with somatostatin not only demonstrates agonistic but also some antagonistic effects on differential signalling cascades. ${ }^{152}$ The clinical significance of these different molecular findings and how they might affect antitumoural efficacy or the development of tachyphylaxis of pasireotid in NET needs further evaluation. In an open-label study in patients with inadequate control of carcinoid syndrome during octreotide LAR, pasireotide improved symptom control in $27 \% .{ }^{138}$ A clinical phase III study of pasireotide LAR versus octreotide LAR is currently recruiting patients with metastatic 
carcinoid tumours whose disease-related symptoms are inadequately controlled by somatostatin analogues (http://ClinicalTrials.gov/ identifier: NCT0069043).

Somatostatin-dopamine chimeras may be another future option in the biotherapy of NET. Dopamine receptors subtype 2 have been reported in a high percentage of low-grade $\mathrm{NET}^{153} 154$ complementing the well-characterised somatostatin receptor subtypes. The somatostatin-dopamine chimera BIM23A760 has been investigated in a phase II clinical study in patients with carcinoid syndrome (http://ClinicalTrials.gov/identifier: NCT01018953), however this trial has been terminated as preliminary study data did not support the expected drug profile.

In comparison with interferon alpha, interferon beta has a much more pronounced antitumoral effect in NET cells in vitro. ${ }^{155} 156$ Interferon beta inhibits the transcription of insulin-like growth factor (IGF) type II and the IGF-IR in neuroendocrine BON1 tumour cells. ${ }^{157}$ This is of interest, because the IGF system plays an essential role in many different cancers including NEC, and most probably contributes to the antitumoural effects of interferon beta. ${ }^{157}$ However, its short half-life currently prevents any clinical utility and effectiveness of interferon beta as an anticancer agent. ${ }^{155}$ Novel strategies such as pegylated interferon beta or interferon beta gene transfer are currently being developed. ${ }^{158}$

\section{CHEMOTHERAPY \\ Chemotherapy of well and moderately differentiated pancreatic NEC}

Chemotherapy is an established therapeutic option in progressive well and moderately differentiated pancreatic NEC (see table 2) and has recently been extensively reviewed. ${ }^{159} 160$ Standard chemotherapy regimens in well and moderately differentiated pancreatic NEC are streptozotocin/doxorubicin and streptozotocin/5-fluorouracil.53 54159161162 With streptozotocin-based regimens partial response rates of $45-65 \%$ have been reported in historical series by Moertel et al (for review see Vilar et al). ${ }^{159}$ An objective tumour response rate of approximately $40 \%$ with a TTP of $9-18$ months has been established in recent studies. ${ }^{161} 164$ Streptozotocin-based protocols are still the most efficient known regimens and are recommended as standard chemotherapy regimens. ${ }^{5354159161162165}$

An alternative established chemotherapy regimen is dacarbazine, ${ }^{159} 162$ with a reported partial response rate of $35 \%$ in first-line chemotherapy of pancreatic NEC. ${ }^{166}$ A combination of 5-fluorouracil, dacarbazine and epirubicine has currently been reported in a small subgroup of 16 patients with pancreatic NEC to produce a partial response rate of up to $58 \%{ }^{167}$

Dacarbazine and temozolomide are each converted to the same active metabolite methyltriazeno-imidazole-carboxamide. ${ }^{168}$ Temozolomide as an oral chemotherapeutic agent therefore was also evaluated in NEC. ${ }^{169-171}$ In NEC of heteroge- nous primary tumour origin (pancreatic, small bowel, bronchial, thymic, others) temozolomide as a single agent caused only a partial tumour response in $5-14 \%$ and a tumour stabilisation in $53-81 \%$, with a median overall TTP of only 7-9 months. ${ }^{169} 171$ Temozolomide-based combination chemotherapies (temozolomide/thalidomide, temozolomide/bevacizumab, others) have been reported in a heterogenous subgroup of 53 patients with pancreatic NET to result in a partial tumour response in $34 \% .{ }^{170}$

Very promising data on temozolomide plus capecitabine have recently been published by Strosberg et al. ${ }^{172}$ In a series of 30 patients with metastatic NEC of the pancreas (66\% with progressive disease, $33 \%$ with newly diagnosed carcinoma) first-line chemotherapy with capecitabine $(750 \mathrm{mg} /$ $\mathrm{m}^{2}$ twice a day, days 1-14) and temozolomide $\left(200 \mathrm{mg} / \mathrm{m}^{2}\right.$ once a day at bedtime, days 10-14) every 28 days was investigated. High efficacy of the regimen was reported, with $70 \%$ partial remissions and $27 \%$ stable disease, as well as a median PFS of 18 months and an estimated overall survival of $92 \%$ at 2 years. ${ }^{172}$ Toxicity mostly consisted of myelosuppression, palmar-plantar erythrodysesthesia, fatigue and nausea. Overall, toxicity was moderate with grade $3 / 4$ toxicities and required dose reductions in only $12 \%$, each. ${ }^{172}$ Temozolomide plus capecitabine thus merits further evaluation in neuroendocrine pancreatic carcinomas in randomised, controlled clinical studies.

The DNA repair enzyme O6-methylguanine DNA methyltransferase (MGMT) contributes to the resistance of tumour cells against temozolomide. ${ }^{168} 173174$ In an immunohistochemical analysis of 97 NET samples (pancreas $n=37$, bronchial carcinoid $n=40$, small intestine carcinoid $n=20$ ) $51 \%$ of pancreatic NET but $0 \%$ of small intestine carcinoid tumours showed MGMT deficiency. ${ }^{170}$ This finding might explain the sensitivity of pancreatic NET but not of small intestine NET against temozolomide. ${ }^{170}$ Whether MGMT deficiency is a predictive biomarker for temozolomide or dacarbazine sensitivity in NEC ${ }^{169} 170$ needs to be addressed in future studies. Sensitising NET cells against temozolomide by depleting intracellular MGMT levels has been discussed as one possible mechanism by which capecitabine might contribute to the antitumoural synergism between capecitabine plus temozolomide. ${ }^{172}$

Oxaliplatin-based regimens also have efficacy and may be considered as salvage chemotherapy in well-differentiated NEC. ${ }^{175}{ }^{176}$ Bajetta et al ${ }^{175}$ reported capecitabine plus oxaliplatin in the XELOX protocol in 27 patients with well-differentiated carcinomas of different primary localisation (lung, pancreas, small intestine, others) inducing $30 \%$ partial response and $48 \%$ stable disease, with a median duration of remission of 20 months and a median survival of 40 months. Preliminary reported data with FOLFOX-6 plus bevazicumab or CAPOX plus bevazicumab merit further investigations. ${ }^{177}$ In contrast, gemcitabine monotherapy ${ }^{178}$ and also gemcitabine-oxaliplatin in the GEMOX protocol ${ }^{176}$ cannot be recommended in NET. 
Table 2 Selected studies on chemotherapy in well-differentiated neuroendocrine carcinomas of the pancreas

\begin{tabular}{|c|c|c|c|c|c|c|}
\hline Authors & Chemotherapy & $\begin{array}{l}\text { Patients } \\
\text { (n) }\end{array}$ & $\begin{array}{l}\text { RR } \\
(\%)\end{array}$ & $\begin{array}{l}\text { SD } \\
(\%)\end{array}$ & $\begin{array}{l}\text { mOS } \\
\text { (months) }\end{array}$ & $\begin{array}{l}\text { Outcome } \\
\text { (months) }\end{array}$ \\
\hline \multirow[t]{2}{*}{ Moertel $1980^{i}$} & $\mathrm{STZ}+5-\mathrm{FU}$ & 42 & 63 & - & 26 & - \\
\hline & STZ & 42 & 36 & - & 16 & - \\
\hline \multirow[t]{2}{*}{ Moertel $1992^{j}$} & $\mathrm{STZ}+\mathrm{DOX}$ & 36 & 69 & - & 26 & - \\
\hline & $\mathrm{STZ}+5-\mathrm{FU}$ & 33 & 45 & - & 17 & - \\
\hline Kouvaraki $2004^{161}$ & $\mathrm{STZ}+\mathrm{DOX}+5-\mathrm{FU}$ & 84 & 39 & - & 37 & PFS 18 \\
\hline Fjallskog $2008^{164}$ & STZ + DOX (liposomal) & 30 & 40 & 57 & $>24$ & TTP 9-11 \\
\hline Ramanathan $2001^{166}$ & Dacarbazin & 50 & 34 & - & 19 & - \\
\hline Ekeblad $2007^{169}$ & Temozolomid & 12 & 8 & 67 & - & - \\
\hline Kulke $2006^{\mathrm{k}}$ & Temozolomid + thalidomid & 11 & 45 & 68 & $>24$ & TTP 13 \\
\hline Kulke $2006^{1}$ & Temozolomid + bevacizumab & 18 & 24 & 70 & - & - \\
\hline Strosberg $2010^{172}$ & Temozolomid + capecitabine & 30 & 70 & 27 & - & PFS 18 \\
\hline Bajetta $2007^{175}$ & Capecitabine + oxaliplatin (XELOX) & 11 & 27 & 45 & 40 & TTP 20 \\
\hline Cassier $2009^{176}$ & Gemcitabine + oxaliplatin (GEMOX) & 18 & 17 & & 23 & TTP 7 \\
\hline Kulke $2004^{178}$ & Gemcitabine & 18 & 0 & 65 & 11 & - \\
\hline
\end{tabular}

Adopted from Vilar et al. ${ }^{15}$

DOX, doxorubicin; 5-FU, 5-fluorouracil; mOS, median overall survival; PFS, progression-free survival; STZ streptozotocine; TTP, time to progression; RR, response rate; SD, stable disease.

\section{Chemotherapy of well and moderately differentiated NEC of the jejunum/ileum}

NEC of the jeiunum/ileum are considered rather insensitive to conventional chemotherapeutics. ${ }^{159}$ Chemotherapy with currently published regimens is not recommended in NEC of the jejunum/ileum according to ENETS guidelines 2008, ${ }^{43}$ NCCN guidelines $2009^{44}$ and NANETS guidelines $2010 .^{45}$ Chemotherapy might only be considered for these tumour entities when all other therapeutic options have already been applied. ${ }^{44}$

In a large series of 249 patients with carcinoid tumours of the small intestine treated with doxorubicine/5-fluorouracil, streptozotocine/5fluorouracil or dacarbazine, Sun et a ${ }^{179}$ reported streptozotocine/5-fluorouracil as the most efficient regimen, with $16 \%$ partial response, $15 \%$ stable disease and a PFS of only 5 months. A recent phase III trial compared chemotherapy using streptozotocin/5-fluorouracil with interferon alpha in 64 patients with progressive, metastatic carcinoid tumours of the small intestine and various other primary tumour locations. ${ }^{146}$ Overall survival in this heterogeneous cohort was not significantly different in both groups, but there was a trend in favour of interferon alpha regarding median PFS. ${ }^{146}$ In the retrospective UKINETS study of 360 patients with liver metastatic midgut carcinoid tumours chemotherapy was a negative prognostic predictor in the univariate analysis. ${ }^{40}$

\section{Chemotherapy of poorly differentiated NEC}

Current guidelines from ENETS and NANETS recommend cisplatin/etoposide as standard chemotherapy in poorly differentiated NEC with high proliferation index Ki-67 greater than 20\%. ${ }^{159} 180181$ Moertel et al ${ }^{163}$ described in 18 patients with poorlydifferentiated NEC using etoposide/cisplatin (etoposide $130 \mathrm{mg} / \mathrm{m}^{2}$ per day on days $1-3$ plus cisplatin $45 \mathrm{mg} / \mathrm{m}^{2}$ per day on days 2 and 3) a complete response in $17 \%$ and a partial response in $50 \%$, resulting in an overall tumour response rate of $67 \%$, with a median survival of 18 months and a median TTP of 11 months. Mitry et al ${ }^{182}$ reported on etoposide/cisplatin (etoposide $100 \mathrm{mg} / \mathrm{m}^{2}$ per day on days $1-3$ plus cisplatin $100 \mathrm{mg} / \mathrm{m}^{2}$ per day on day 1) in 41 patients with poorly differentiated NEC complete response in $9.5 \%$ and partial response in $32 \%$. This resulted in an overall tumour response rate of $41.5 \%$ with a median overall survival of 15 months, a median PFS of 8.9 months and a 2 -year survival of less than $20 \% .{ }^{182}$ Hainsworth et al ${ }^{183}$ treated 78 patients with poorly differentiated NEC with paclitaxel/carboplatin/etoposide. They found a complete response in $15 \%$ and partial response in $38 \%$, resulting in an overall tumour response rate of $53 \%$ with 2-year and 3-year survival rates of 33\% and $24 \%$, respectively. Paclitaxel/carboplatin/ etoposide did not show superior efficacy but a much higher toxicity profile compared with the standard etoposide/cisplatin.

\section{Novel targeted therapies}

Novel targeted therapies in oncology aim to target growth factors, growth factor receptors, receptor tyrosine kinases, or intracellular signalling cascades in order to effect tumour angiogenesis and/or tumour cell growth and apoptosis by inactivation of overexpressed or overactivated signalling cascades. NEC are hypervascularised tumours that express growth factors and their respective receptors to a high percentage, for example vascular endothelial growth factor receptor (VEGFR), epidermal growth factor receptor (EGFR), platelet derived growth factor receptor (PDGFR), as well as insulin-like growth factor-1 receptor (IGF-1R). ${ }^{184}$ The phophoinositol-3-kinase (PI(3)K)-Akt-mTOR and the Ras-Raf-MEK-Erk1/2 pathway are key regulators of cell survival and proliferation, activated by various growth factors. Growth factors and their receptors, as well as the essential $\mathrm{PI}(3) \mathrm{K} / \mathrm{Akt} /$ mTOR and the Ras/Raf/Mek/Erk intracellular signalling cascade, ${ }^{184}$ are thus potential targets of novel targeted therapeutic strategies. See figure 1.

Results for novel targeted therapies from phase II clinical studies in NET are available with bevacizumab, ${ }^{185}$ sunitinib, ${ }^{186}$ everolimus, ${ }^{187} 188$ temsirolimus, ${ }^{189}$ imatinib, ${ }^{190}$ sorafenib and others, as has been summarised in current reviews. ${ }^{184} 191-193$

\section{Bevacizumab}

Angiogenesis inhibition by targeting VEGF is an established strategy in oncology and is currently widely investigated in clinical trials in various malignancies, ${ }^{194} 195$ while high VEGF expression is a negative prognostic factor in various malignancies. ${ }^{196} 197$ In patients with NEC ${ }^{198} 199$ high VEGF expression has also been reported to be a negative prognostic factor associated with increased microvessel density, higher incidence of metastasis and tumour progression as well as shorter median PFS. ${ }^{198} 199$ Bevacizumab, a monoclonal antibody against VEGF, significantly decreased tumour blood flow in patients with NET. ${ }^{185}$ A prospective randomised clinical phase III study of octreotide LAR plus bevacizumab versus octreotide LAR plus interferon alpha in advanced carcinoid patients 


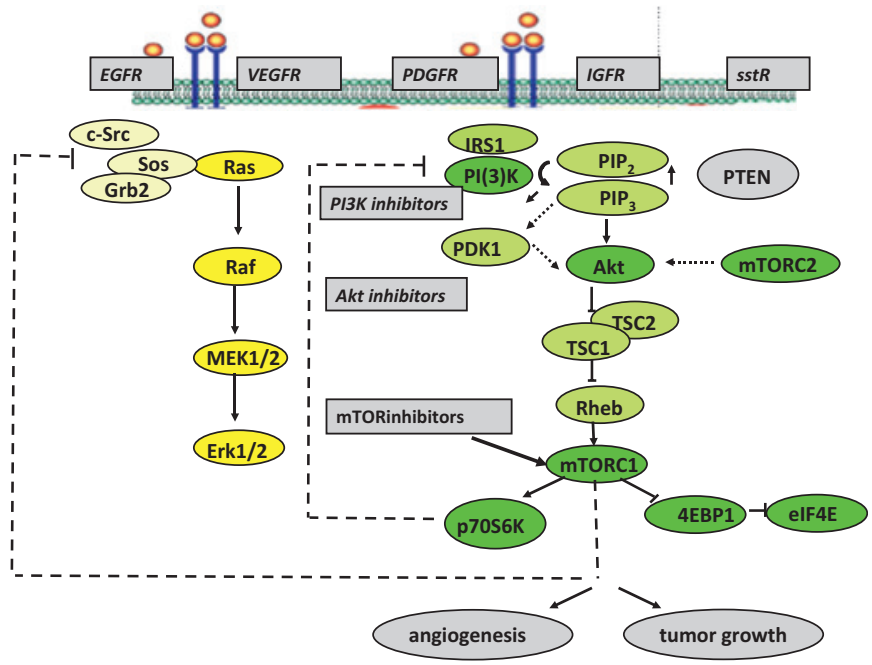

Figure 1 Diagram of phophoinositol-3-kinase (PI(3)K)-Akt-mammalian target of rapamycin (mTOR) and Ras-Raf-MEK-Erk signalling cascades and novel targeted therapy in neuroendocrine carcinomas. EGFR, epidermal growth factor receptor; IGFR, insulin-like growth factor receptor; IRS, insulin receptor substrate; PDGFR, plateletderived growth factor receptor; $\mathrm{PDK}_{1}$, phosphoinositide-dependent kinase 1; $\mathrm{PIP}_{2}$, phosphatidylinositol-4,5-bisphosphate; $\mathrm{PIP}_{3}$, phosphatidylinositol-3,4,5-trisphosphate; PTEN, phosphatase and tensin homologue; TSC, tuberous sclerosis; VEGFR, vascular endothelial growth factor receptor.

(http://ClinicalTrials.gov/ identifier: NCT00569127), as well as phase II clinical studies of temozolomide plus bevacizumab (http://ClinicalTrials.gov/ identifier: NCT00137774), CAPOX plus bevacizumab (http://ClinicalTrials.gov/ identifier: NCT00398320), FOLFOX plus bevacizumab (http://ClinicalTrials. gov/ identifier: NCT00227617) and RAD001 plus bevacizumab (http://ClinicalTrials.gov/ identifier: NCT00607113) are still ongoing.

\section{Sunitinib}

Promising preliminary data of a placebo-controlled phase III clinical study in NEC of the pancreas have recently been presented for sunitinib. Sunitinib (SU-11248) is a multikinase inhibitor with activity against VEGFR-1, VEGFR-2, VEGFR-3, plateletderived growth factor receptor, c-Kit and Flt-3, which is currently approved in renal cell carcinoma and gastrointestinal stromal tumours. In a preclinical model of islet cell tumours in RIP1-Tag2 mice, sunitinib has demonstrated antitumour efficacy. ${ }^{200}$ Kulke et al ${ }^{186}$ investigated sunitinib in a clinical phase II study in 106 patients with NEC.

In a prospective randomised clinical phase III study of sunitinib versus placebo in advanced pancreatic NEC patients (http://ClinicalTrials.gov/ identifier: NCT00428597) patients received either sunitinib $37.5 \mathrm{mg} /$ day continuous daily dosing $(n=75)$ or placebo $(n=79) \cdot{ }^{201-203}$ This trial was halted prematurely due to sunitinib significantly prolonging PFS compared with placebo. ${ }^{201-203}$ According to preliminary data presentations, ${ }^{201-203}$ PFS was 11.4 months in the sunitinib group versus 5.5 months in the placebo group (HR 0.418, $\mathrm{p}=0.0001) .{ }^{201-203}$ Median overall survival was not reached, but sunitinib was favoured by a HR of 0.409 ( $p=0.0204)^{201-203}$ The Committee for Medicinal Products for Human Use adopted sunitinib as being indicated for the treatment of unresectable or metastatic, well-differentiated pancreatic NET with disease progression in adults. Experience with sunitinib as first-line treatment is limited. The European Medicines Agency (EMA) approved this new indication in 11/2010 and the European Public Assessment Report is available (http://www.ema.europa.eu). Common side-effects of sunitinib include fatigue, asthenia, diarrhoea, nausea, vomiting, anorexia, bleeding complications, mucosal inflammation, hand-foot skin reaction, hypertension, anaemia, granulocytopaenia and thrombocytopaenia. ${ }^{204}$ Another side-effect of sunitinib that clinicans should also be aware of is hypothyroidism. ${ }^{204}$

\section{Everolimus}

The PI(3)K-Akt-mTOR pathway is key regulator of cell survival and proliferation initiated by growth factors and their activating receptor tyrosine kinases. Activation of PI(3)K is followed by downstream phosphorylation of Akt and mTOR. ${ }^{205-208}$ Constitutive activation of the PI(3)K-Akt-mTOR pathway is present in many malignancies including NEC. In NEC a loss or downregulation of the expression of tumour suppressor phosphatase and tensin homologue 209210 or of tuberous sclerosis $2^{210}$ resulting in overactivation of the $\mathrm{PI}(3) \mathrm{K}-\mathrm{Akt}-\mathrm{mTOR}$ pathway, as well as the expression of activated Akt ${ }^{211}$ and the expression of activated $\mathrm{mTOR}^{212}$ has been described in a high percentage of cases. The rapamycine analogues everolimus and temsirolimus are mTORC-1 complex inhibitors, which are currently approved in renal cell carcinoma. ${ }^{213}$ Preclinical in-vitro and in-vivo data in several NET models have demonstrated that inhibition of the PI (3)K-Akt-mTOR signalling cascade by the mTOR inhibitors rapamycine $e^{214215}$ and everolimus ${ }^{184} 216$ inhibits tumour cell growth and induces apoptosis. Clinical phase II studies of mTOR inhibitors in NEC of pancreatic and midgut origin have seen antitumoural efficacy of temsirolimus ${ }^{189}$ and of everolimus. ${ }^{187} 188$ In addition, in malignant insulinomas fast and efficient glycaemic control by rapamycin or everolimus ${ }^{217} 218$ has been reported in several case reports.

In a prospective randomised clinical phase III study of everolimus plus best supportive care versus placebo plus best supportive care in advanced pancreatic NEC (RADIANT-3 Study; http://Clinical Trials.gov/ identifier: NCT00510068) patients received either everolimus $10.0 \mathrm{mg} /$ day continous daily dosing $(n=207)$ or placebo $(n=203) .{ }^{219} 220$ According to preliminary data presentations, ${ }^{219} 220$ there was a 2.4-fold prolongation in median PFS with 11.0 months in the everolimus group versus 4.6 months in the placebo group (HR 0.35, $\mathrm{p}=0.0001) .{ }^{219} 220$ Prolonged benefit in a subset of patients has been suggested by estimates for 18 months PFS rates being 34\% (95\% CI 26\% to $43 \%)$ in the everolimus group versus $9 \%(95 \% \mathrm{CI}$ $4 \%$ to $16 \%$ ) in the placebo group. ${ }^{220}$

In another prospective randomised clinical phase III study of everolimus plus octreotide LAR in advanced carcinoid tumours of various origin 
(RADIANT-2 Study; http://ClinicalTrials.gov/ identifier: NCT00412061) has been investigated. ${ }^{221}$ Patients received either everolimus $10.0 \mathrm{mg} /$ day continuous daily dosing plus octreotide LAR $30 \mathrm{mg}$ intramuscularly every 28 days $(n=216)$ or placebo plus octreotide LAR $30 \mathrm{mg}$ intramuscularly every 28 days $(n=213) .^{221}$ According to a preliminary data presentation, ${ }^{221}$ there was a moderate prolongation in median PFS of 16.4 months in the

\section{Summary}

This review discusses current standards as well as novel therapeutic strategies in advanced NEC of midgut and pancreatic origin. Especially focused issues are:

- Recent comprehensive guidelines for the diagnosis and treatment of gastroenteropancreatic NET have been published by ENETS, NCCN and NANETS.

- It is important to consider prognostic parameters that are also useful for therapeutic decision-making. Recommended prognostic parameters for such purposes are differing tumour biologies of NET of the jejunum/ileum and of pancreatic origin, different tumour gradings including proliferation index Ki-67, the presence or absence of distant metastases, as well as the pattern and tumour load of hepatic metastases.

- Surgery of well-differentiated NEC is recommended in local/regional disease but also at distant disease with liver metastases. Resection of the primary seems feasible despite liver metastases and may be beneficial especially in NET of the jejunum/ileum, while currently not generally recommended in panreatic NET. Resection of liver metastases or debulking more than $90 \%$ of liver metastases is recommended and may improve overall survival.

- Neoadjuvant and adjuvant therapeutic strategies for liver surgery of hepatic metastasised NEC are urgently needed. Further research should address this issue.

- In well-differentiated NET of the jejunum/ileum, octreotide LAR in a randomised placebo-controlled study caused significantly longer time to tumour progression. Somatostatin analogues are thus no longer a standard for symptom control in functionally active tumours, but may also be considered for antiproliferative treatment in well-differentiated functional and non-functional NET of the jejunum/ileum. Results of another randomised placebo-controlled study of lanreotide autogel in enteropancreatic NET are expected in 2011.

- Streptozotocin-based regimens are still the recommended chemotherapy standard in well-differentiated pancreatic NET. Temozolomide plus capecitabine may be a novel promising strategy, which merits further evaluation. Preliminary data on oxaliplatin-based regimens (eg, XELOX, CAPOX, FOLFOX) as second-line chemotherapy are promising, but further studies are needed.

- SIRT with 90Y-microspheres is a novel local-ablative strategy in disseminated liver metastases. Tumour response with SIRT in NEC is comparable to results with repetitive treatment cycles of TAE or TACE. All these local ablative methods are recommended in inoperable hepatic metastases.

- PRRT with 90Yttrium-DOTA-TOC, 90Yttrium-DOTA-TATE and 177LutetiumDOTA-TATE is a promising strategy in distant metastases of NEC. PRRT seems indicated for patients with inoperable extrahepatic with or without intrahepatic tumour load with sufficient expression of somatostatin receptors.

- Several novel targeted therapies are currently being investigated in well and moderately differentiated NEC. Everolimus and sunitinib have both demonstrated improved PFS against placebo in current clinical phase III trials in NEC of the pancreas. The European Medicines Agency (EMA) approved sunitinib for this new indication in $11 / 2010$. Approval of everolimus for this new indication is currently still pending but may be expected in the near future.

- Individual multimodal therapeutic strategies in patients with NEC require interdisciplinary decision-making in an institutionalised tumour board. Patients should be treated in randomised controlled studies at specialised tertiary care centres. everolimus plus octreotide LAR group versus 11.3 months in the placebo plus ocreotide LAR group (HR 0.77 ; 95\% CI 0.59 to $1.00, \mathrm{p}=0.026$, which failed the prespecified significance level of $\mathrm{p}=0.0246) .{ }^{219}$ However, several data imbalances may have favoured the placebo group. ${ }^{221}$ The final data analysis and publication of this trial has to be awaited. Approval of everolimus for NEC is currently pending but may be expected in the near future. Common side-effects of everolimus known from approved indications and the current phase III trials include stomatitis, rash, diarrhoea, fatigue, infections, non-infectious pneumonitis, anaemia, lymphopaenia, hypercholesterinaemia, hyperlipidaemia and hyperglycaemia. ${ }^{220-222}$

Tumours may escape mTOR inhibition by paradoxical activation of survival signalling pathways. $^{223}$ Possible strategies to overcome mTOR escape are dual blockade strategies. Accordingly, current clinical phase II studies in NEC are sorafenib and everolimus (http://ClinicalTrials.gov/ identifier: NCT00942682), bevacizumab and everolimus (http://ClinicalTrials.gov/ identifier: NCT00607113) and erlotinib and everolimus (http://ClinicalTrials.gov/ identifier: NCT00843531).

\section{IGF-IR inhibition}

Various further novel strategies of targeted therapy in NEC are under development. For example, inhibition of the IGF-I-IGF-IR system decreases cell growth and promotes apoptosis in various human NET cell lines ${ }^{216} 224$ as well as tumour control in a xenograft model. ${ }^{225}$ Several IGF-1R antibodies and IGF-1R tyrosine kinase inhibitors are currently in clinical development for many different types of cancer. $^{224}$ Potential problems of in-vivo resistance to IGF-1R inhibition need further evaluation and caution. $^{226}$ The monoclonal antibodies against IGF-IR AMG479 (http://ClinicalTrials.gov/ identifier: NCT01024387), IMC-A12 (http://ClinicalTrials. gov/ identifier: NCT00781911) and MK-0646 (http://ClinicalTrials.gov/ identifier: NCT00610129) are currently in clinical phase II studies in patients with metastatic NEC.

\section{How to choose the appropriate sequence of therapeutic options in inoperable carcinomas?}

Prognostic factors of NEC may be helpful for clinical decisions and the determination of a therapeutic strategy. Inoperable, well-differentiated NEC with minor/moderate tumour load in an asymptomatic patient might allow an initial watch-andwait strategy with restaging every 3 months to evaluate tumour growth dynamics. ${ }^{44}$ However, in most cases the 'wait-and-see' strategy will result in tumour progression as demonstrated by the PROMID study with a median TTP of only 6.0 months in the placebo group in therapy-naive midgut NEC. ${ }^{35}$ The watch-and-wait strategy needs a critical re-evaluation. On the other hand, a high initial tumour load, a symptomatic patient, a higher proliferation index $\mathrm{Ki}-67$ or a progressive disease will necessitate initial palliative therapy. ${ }^{44}$ Evidence-based data on therapeutic sequence 
strategies in inoperable metastasised NEC are not available at present. Consensus recommendations on possible therapeutic strategies and sequences are provided by the recently published ENETS guidelines, ${ }^{27} 43 \quad 96144162 \quad 227-237$ NCCN guidelines, ${ }^{44}$ NANETS guidelines, ${ }^{45} 54$ 238-242 European Society for Medical Oncology (ESMO) Clinical Practice Guidelines $^{243}$ and Nordic guidelines. ${ }^{165}$

Acknowledgement This manuscript has been in part supported by a grant from the German Federal Ministry of Education and Research (01EX1021B, Spitzencluster M4, Verbund Personalisierte Medizin: Teilprojekt NeoExNET (PM1)).

References The full reference list is available online only. To view this file please visit the journal online (http://gut.bmj.com).
Competing interests CJA has received research contracts (Ipsen, Novartis), lecture honorarium (Ipsen, Novartis, Pfizer) and advisory board honorarium (Ipsen, Novartis). BG declares no competing interests.

Provenance and peer review Not commissioned; externally peer reviewed.

Amendment After this manuscript was accepted for publication, the clinical phase 3 study of sunitinib in patients with neuroendocrine tumors of the pancreas and the clinical phase 3 study of everolimus in patients with neuroendocrine tumors of the pancreas were both been published as follows:

Raymond E, Dahan L, Raoul JL, et al. Sunitinib malate for the treatment of pancreatic neuroendocrine tumors. N Engl J Med 2011;364:501-13.

Yao JC, Shah MH, Ito T, et al. Everolimus for advanced pancreatic neuroendocrine tumors. N Engl J Med 2011;364:514-23.

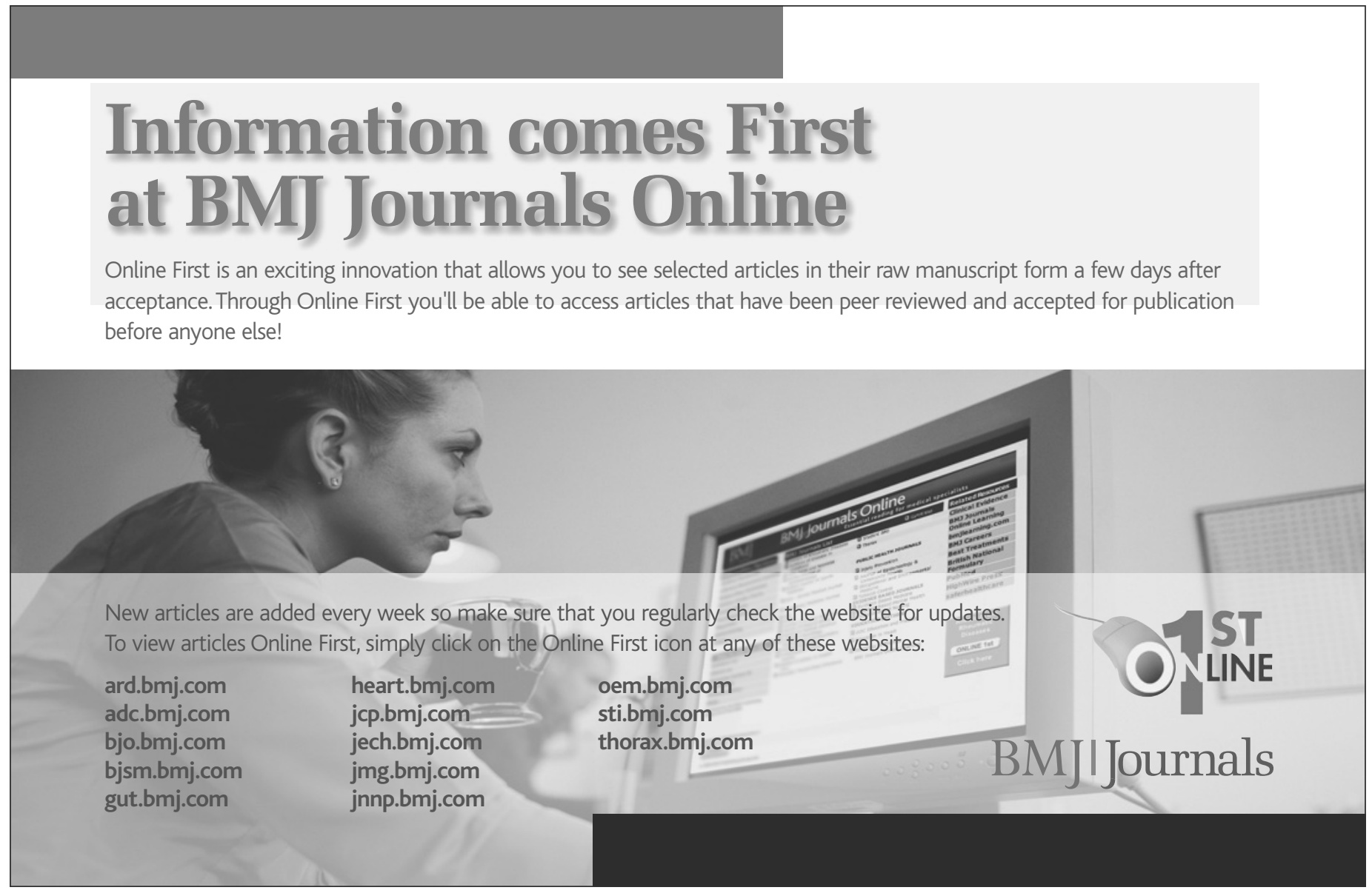




\section{GUT}

Therapeutic strategies for advanced neuroendocrine carcinomas of jejunum/ileum and pancreatic origin

Christoph J Auernhammer and Burkhard Göke

Gut 2011 60: 1009-1021 originally published online March 4, 2011

doi: $10.1136 /$ gut.2009.204453

Updated information and services can be found at:

http://gut.bmj.com/content/60/7/1009

Supplementary Material

References

Email alerting service
Supplementary material can be found at:

http://gut.bmj.com/content/suppl/2011/05/25/gut.2009.204453.DC1.ht $\mathrm{ml}$

\section{These include:}

This article cites 240 articles, 54 of which you can access for free at: http://gut.bmj.com/content/60/7/1009\#BIBL

Receive free email alerts when new articles cite this article. Sign up in the box at the top right corner of the online article.

Collections

Topic Articles on similar topics can be found in the following collections

Gut Education (56)

GUT Recent advances in clinical practice (71)

Hepatic cancer (436)

Pancreas and biliary tract (1899)

Gastrointestinal hormones (842)

\section{Notes}

To request permissions go to:

http://group.bmj.com/group/rights-licensing/permissions

To order reprints go to:

http://journals.bmj.com/cgi/reprintform

To subscribe to BMJ go to:

http://group.bmj.com/subscribe/ 